\title{
Alterations in Skeletal Muscle Protein-Tyrosine Phosphatase Activity and Expression in Insulin-resistant Human Obesity and Diabetes
}

\author{
Faiyaz Ahmad, ${ }^{\star}$ John L. Azevedo, Jr., ${ }^{\ddagger}$ Ronald Cortright, ${ }^{\ddagger}$ G. Lynis Dohm, ${ }^{\ddagger}$ and Barry J. Goldstein* \\ *Dorrance H. Hamilton Research Laboratories, Division of Endocrinology, Diabetes and Metabolic Diseases, Department of Medicine, \\ Jefferson Medical College of Thomas Jefferson University, Philadelphia, Pennsylvania 19107; and ${ }^{\ddagger}$ Department of Biochemistry, East \\ Carolina University School of Medicine, Greenville, North Carolina 27858
}

\begin{abstract}
Obese human subjects have increased protein-tyrosine phosphatase (PTPase) activity in adipose tissue that can dephosphorylate and inactivate the insulin receptor kinase. To extend these findings to skeletal muscle, we measured PTPase activity in the skeletal muscle particulate fraction and cytosol from a series of lean controls, insulin-resistant obese (body mass index $>30$ ) nondiabetic subjects, and obese individuals with non-insulin-dependent diabetes. PTPase activities in subcellular fractions from the nondiabetic obese subjects were increased to $140-170 \%$ of the level in lean controls $(P<0.05)$. In contrast, PTPase activity in both fractions from the obese subjects with non-insulin-dependent diabetes was significantly decreased to $39 \%$ of the level in controls $(P<0.05)$. By immunoblot analysis, leukocyte antigen related (LAR) and protein-tyrosine phosphatase $1 \mathrm{~B}$ had the greatest increase (threefold) in the particulate fraction from obese, nondiabetic subjects, and immunodepletion of this fraction using an affinity-purified antibody directed at the cytoplasmic domain of leukocyte antigen related normalized the PTPase activity when compared to the activity from control subjects. These findings provide further support for negative regulation of insulin action by specific PTPases in the pathogenesis of insulin resistance in human obesity, while other regulatory mechanisms may be operative in the diabetic state. (J. Clin. Invest. 1997. 100:449458.) Key words: insulin receptor • tyrosine kinase $\cdot$ signal transduction $\bullet$ insulin resistance $\bullet$ protein phosphatase
\end{abstract}

\section{Introduction}

Resistance to the biological action of insulin in its target tissues is a major feature of the pathophysiology of human obesity and non-insulin-dependent diabetes mellitus (NIDDM) $)^{1}(1-3)$. Recent insights into the mechanism of insulin action have demonstrated that reversible tyrosine phosphorylation of the insu-

Address correspondence to Barry J. Goldstein, M.D., Ph.D., Director, Division of Endocrinology, Diabetes and Metabolic Diseases, Jefferson Medical College, Room 349, Alumni Hall, 1020 Locust Street, Philadelphia, PA 19107. Phone: 215-503-1272; FAX: 215-9237932; E-mail: b_goldstein@lac.jci.tju.edu

Received for publication 21 November 1996 and accepted in revised form 10 April 1997.

J. Clin. Invest.

(C) The American Society for Clinical Investigation, Inc.

0021-9738/97/07/0449/10 \$2.00

Volume 100, Number 2, July 1997, 449-458 lin receptor and its cellular substrate proteins plays a central role in the mechanism of insulin action (4), although it is still not clear how these events are regulated and what cellular and molecular defects may occur that cause the insulin resistance observed in disease states.

Several recent studies have provided evidence that protein-tyrosine phosphatases (PTPases) have an important role in the regulation of insulin signal transduction (5). Since the autophosphorylated insulin receptor does not self-dephosphorylate, cellular PTPases are required to dephosphorylate the receptor and attenuate the activity of the autophosphorylated insulin receptor tyrosine kinase. In addition, PTPases can modulate postreceptor signaling by dephosphorylating cellular substrate proteins for the insulin receptor such as IRS-1 and Shc, which signal to downstream enzymes as docking proteins that bind a number of src-homology 2 (SH2) domain-containing proteins, and activate their signaling capacity (4). Thus, the opposing effects of phosphorylation by the insulin receptor kinase, and dephosphorylation by cellular PTPases, will also balance the tyrosine phosphorylation state of postreceptor proteins in the insulin action pathway, and will regulate their downstream signaling potential.

From the expanding superfamily of protein-tyrosine phosphatases (6), work in our laboratory and others has implicated the tandem-domain transmembrane enzymes leukocyte antigen related (LAR) (7) and leukocyte common antigen-related phosphatase (LRP)/RPTP- $\alpha$ (8), and the intracellular, singledomain enzymes PTP1B $(9,10)$ and SH-PTP2/syp (11), as candidate PTPases for the regulation of the insulin action pathway. In addition to being relatively prominent PTPases in insulin-sensitive tissues, these enzymes have catalytic activity towards the insulin receptor or its substrate proteins in studies done both in vitro and in vivo $(5,12)$. Furthermore, studies in cell systems have provided evidence that LAR, LRP, and PTP1B can serve as negative regulators of insulin receptor phosphorylation (13-19), while SH-PTP2 appears to be a positive mediator of distal insulin signaling (20-22).

In addition to the role for PTPases in the steady-state regulation of cellular insulin action in normal physiology, we and others have postulated that an elevation of specific PTPases might be an etiologic factor in the tissue insulin resistance that occurs in human obesity and other disease states, including NIDDM. In studies testing this hypothesis, we have found recently that adipose tissue from obese human subjects con-

1. Abbreviations used in this paper: BMI, body mass index; LAR, leukocyte antigen related; LRP, leukocyte common antigen-related phosphatase; NIDDM, non-insulin-dependent diabetes mellitus; PTPase, protein-tyrosine phosphatase; RCM, reduced, carboxamidomethylated and maleyated; SH, src-homology. 
tained increased PTPase activity towards the insulin receptor that was primarily due to an increase in the abundance of the LAR PTPase (23). In a follow-up study, we also provided evidence that the increased PTPase activity in the obese subjects appeared to be reversible, with a significant decrease in the tissue PTPase activity as well as the abundance of both LAR and PTP1B in a series of obese subjects after a sustained loss of $10 \%$ of their body weight (24).

The present work extends these findings to skeletal muscle to explore whether changes in PTPases in this tissue are also associated with insulin resistance. This is of importance because skeletal muscle is regarded to be the major site of tissue insulin resistance in obesity and diabetes with respect to glucose disposal (25). We examined PTPase activity towards the insulin receptor and the abundance of specific PTPase enzymes (LAR, LRP, PTP1B, and SH-PTP2) in a series of lean and nondiabetic obese subjects as well as obese individuals with NIDDM. We found a strong association between body mass index (BMI) and PTPase activity towards the insulin receptor in skeletal muscle from nondiabetic obese subjects, and immunoblot analysis showed that the abundance of several candidate PTPases was increased. Immunodepletion of the muscle protein lysate with antibodies to LAR normalized the increased PTPase activity, suggesting that LAR and/or a related transmembrane PTPase enzyme play(s) an important role in the augmented PTPase activity observed in obesity. Interestingly, in the obese subjects with diabetes, PTPase activity and the abundance of several PTPase enzymes was significantly decreased, suggesting that additional regulatory mechanisms are apparent in the diabetic state.

\section{Methods}

Human subjects. The experimental protocol was approved by the East Carolina University Policy and Review Committee on Human Research, and informed consent was obtained from all patients. Patients were categorized as lean, obese nondiabetic, or obese NIDDM according to BMI (obese if BMI $>30 \mathrm{~kg} / \mathrm{m}^{2}$ ) and National Diabetes Data Group Criteria (26). At the time the tissue sample was obtained, only one of the diabetic subjects had received any insulin therapy for an elevated glucose level. Oral medications for diabetes were withheld before the surgical procedure. Each of the lean subjects and two of the obese subjects were undergoing total abdominal hysterectomy for benign conditions; three of the obese subjects and all of the diabetic individuals were undergoing gastric bypass surgery.

Muscle biopsy. Muscle biopsies were obtained from elective abdominal surgical procedures on female patients. Surgery was performed on the patients after an overnight fast. General anesthesia was initiated with a short-acting barbiturate, and was maintained with fentanyl and nitrous oxide/oxygen inhalation. A $3 \times 2 \times 0.5$-cm muscle biopsy was obtained from the rectus abdominus muscle after entry into the abdominal cavity. A portion of the muscle was snap-frozen in liquid nitrogen and stored at $-85^{\circ} \mathrm{C}$ after a sample was taken for analysis of glucose transport, as indicated below.

Glucose and insulin assay. Blood samples for insulin and glucose levels were taken intraoperatively. Plasma glucose was analyzed by the glucose oxidase method (glucose analyzer II; Beckman Instruments, Inc., Fullerton, CA). Insulin was measured using an automated microparticle enzyme immunoassay (IMX analyzer; Abbott Laboratories Diagnostic Division, Abbott Park, IL).

Glucose transport into skeletal muscle strips. Immediately after removal, a portion of each freshly isolated muscle sample was transported to the laboratory in an airtight container with oxygenated Krebs-Henseleit buffer. Muscle strips weighing $\sim 25 \mathrm{mg}$ were teased from the sample, and insulin-stimulated transport of 2-deoxyglucose was measured using an incubation system and methods described previously (27).

Preparation of skeletal muscle homogenates. Approximately 200 $\mathrm{mg}$ of frozen powdered skeletal muscle from each patient was homogenized in $2 \mathrm{ml}$ of ice-cold buffer containing $1 \mathrm{mM} \mathrm{DTT}, 4 \mathrm{mM}$ EDTA, $2.5 \mathrm{mM}$ benzamidine, $1 \mu \mathrm{M}$ leupeptin, $1 \mu \mathrm{M}$ pepstatin, $0.15 \mu \mathrm{M}$ aprotinin, and $2 \mathrm{mM}$ PMSF in $25 \mathrm{mM}$ Hepes, $\mathrm{pH}$ 7.4, with eight up and down strokes (Polytron; Brinkman Instruments, Westbury, NY) at a setting of seven. The crude homogenate was centrifuged at $350,000 \mathrm{~g}$ for $30 \mathrm{~min}$, and the resulting supernatant was taken as the soluble cytosol fraction. Proteins in the particulate fraction were then solubilized by treating the pellet with ice-cold buffer A containing $1 \%$ (vol/vol) Triton X-100 and $0.6 \mathrm{M} \mathrm{KCl}$, stirring for 45 min at $4^{\circ} \mathrm{C}$, and then centrifuging at $150,000 \mathrm{~g}$ for $1 \mathrm{~h}$. The resulting supernatant was dialyzed against the homogenization buffer overnight. Previous work in rat skeletal muscle showed that the portion of the particulate fraction that was insoluble in Triton X-100 and $0.6 \mathrm{M}$ $\mathrm{KCl}$ lacked detectable expression of LAR, LRP, PTP1B, and SHPTP2 by immunoblotting, and was not studied further in the present work (Ahmad, F., and B.J. Goldstein, unpublished observations). Protein was assayed by the method of Bradford (28), and the tissue fractions were diluted to $1 \mu \mathrm{g}$ protein per $\mu \mathrm{l}$ before use.

FPLC analysis of skeletal muscle PTPases. Protein extracted from the solubilized particulate fraction was dialyzed against $10 \mathrm{mM}$ imidazole- $\mathrm{HCl}$ buffer, pH 7.0, containing $50 \mathrm{mM} \mathrm{NaCl}, 2$ mM EDTA, $1 \mathrm{mM}$ benzamidine, $2 \mathrm{mM}$ PMSF, $0.1 \%$ (vol/vol) $\beta$-mercaptoethanol, and $10 \%$ (vol/vol) glycerol. Samples of $2 \mathrm{ml}$, containing $7.5 \mathrm{mg}$ protein, were applied to Superose 6-12-12 fast protein liquid chromatography (FPLC) columns (Pharmacia LKB Biotechnology Inc., Piscataway, $\mathrm{NJ})$ set in series, and chromatography was performed in the dialysis buffer at a flow rate of $0.3 \mathrm{ml}$ per min. Fractions of $1 \mathrm{ml}$ were collected, and $50 \mu \mathrm{l}$ of each fraction was assayed for PTPase activity with RCM-lysozyme as substrate. Columns were calibrated with myosin, BSA, ovalbumin, and lysozyme protein size standards.

Assay of tissue PTPase activity with autophosphorylated insulin holoreceptors as substrate. Partially purified insulin holoreceptors were obtained by wheat germ agglutinin agarose chromatography (29) of solubilized plasma membranes from Chinese hamster ovary (CHO) cells overexpressing the recombinant human insulin receptor (30). Aliquots of $4 \mathrm{mg}$ protein were autophosphorylated in a $0.45-\mathrm{ml}$ reaction containing $1 \mathrm{mM}$ insulin, $5 \mathrm{mM} \mathrm{MnCl}_{2}, 0.1 \mathrm{mM} \mathrm{ATP}, 180 \mu \mathrm{Ci}$ of $\gamma$ - $\left[{ }^{32} \mathrm{P}\right] \mathrm{ATP}(3,000 \mathrm{Ci} / \mathrm{mmol})$ and $0.1 \%(\mathrm{vol} / \mathrm{vol})$ Triton X-100 in $50 \mathrm{mM}$ Hepes buffer, $\mathrm{pH} 7.6$, at $4^{\circ} \mathrm{C}$ for $120 \mathrm{~min}$, and unincorporated $\left[{ }^{32} \mathrm{P}\right] \mathrm{ATP}$ was removed (Bio-Gel P6 spin column; Bio-Rad Laboratories, Richmond, CA). Aliquots of $25 \mu \mathrm{l}$ of the labeled receptors were incubated with $40 \mu \mathrm{l}$ of solubilized human muscle particulate enzymes in a $125-\mu \mathrm{l}$ reaction containing $1 \mathrm{mM}$ DTT and $2 \mathrm{mM}$ EDTA

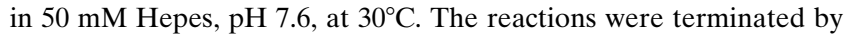
the addition of $0.5 \mathrm{ml}$ of ice-cold stop solution containing $10 \mathrm{mM}$ ATP, $10 \mathrm{mM}$ sodium pyrophosphate, $4 \mathrm{mM}$ EDTA, $100 \mathrm{mM} \mathrm{NaF}$, $2 \mathrm{mM}$ sodium vanadate, $0.1 \mathrm{mg} / \mathrm{ml}$ aprotinin, and $2 \mathrm{mM}$ PMSF in $50 \mathrm{mM}$ Hepes buffer, $\mathrm{pH}$ 7.6. After boiling in gel sample buffer containing $100 \mathrm{mM}$ DTT, aliquots were subjected to electrophoresis in gel containing SDS and $7.5 \%(\mathrm{wt} / \mathrm{vol})$ polyacrylamide (31). Dephosphorylation of the $95-\mathrm{kD} \beta$-subunit of the insulin receptor was analyzed by PhosphorImager analysis of the dried gel (Molecular Dynamics Inc., Sunnyvale, CA).

Reduced, carboxamidomethylated, and maleyated (RCM)-lysozyme as PTPase substrate. For use as a PTPase substrate, RCM-lysozyme was tyrosine phosphorylated as described (32) using insulin receptors purified from the CHO-hIR cells. PTPase activity was assayed using $20 \mu \mathrm{l}$ of the indicated tissue fraction diluted to $<1 \mathrm{U} / \mathrm{ml}$ in buffer, and preincubated for $5 \mathrm{~min}$ at $30^{\circ} \mathrm{C}$. The reaction was initiated by the addition of $20 \mu \mathrm{l}$ of phosphotyrosyl RCM-lysozyme $(10 \mathrm{mM})$. The reaction was terminated with the addition of $0.9 \mathrm{ml}$ of acidic charcoal mixture $(0.9 \mathrm{M} \mathrm{NaCl}, 90 \mathrm{mM}$ sodium pyrophosphate, $2 \mathrm{mM}$ $\mathrm{NaH}_{2} \mathrm{PO}_{4}, 4 \%$ [vol/vol] Norit A; Sigma Chemical Co., St. Louis, 
$\mathrm{MO})$. After centrifugation in microfuge for $1 \mathrm{~min}$, the amount of radioactivity in $0.4 \mathrm{ml}$ of supernatant was measured by Cerenkov counting in a liquid scintillation counter. $1 \mathrm{U}$ of PTPase activity was defined as the amount releasing $1 \mathrm{nmol}$ of phosphate per min.

Immunoblot analysis of PTPase abundance. Aliquots of the skeletal muscle particulate and cytosol fractions (40 $\mu \mathrm{g}$ protein) were fractionated on SDS-polyacrylamide gels, transferred to nitrocellulose filters, and immunoblotted with antibodies to LAR, LRP, PTP1B, and SH-PTP2 as described in our previous work (23). Immunoreactive proteins were visualized and quantitated by PhosphorImager analysis.

Immunodepletion studies with PTPase antibodies. Aliquots of solubilized skeletal muscle particulate fractions from the lean, obese, and diabetic subjects were incubated in separate experiments with excess antibody to individual or a mix of the four PTPases (LAR, LRP, PTP-1B, and SH-PTP2), and the complexed PTPases were precipitated (Trisacryl protein-A beads; Pierce, Rockford, IL) to deplete the supernatant of the enzymes. Normal rabbit IgG was used as control. Experiments performed in parallel with recombinant LAR protein determined that $4 \mu \mathrm{g}$ of affinity-purified LAR antibody provided $\sim 100$-fold excess of antibody that resulted in depletion of $90-95 \%$ of the LAR enzyme from $30 \mu \mathrm{g}$ of homogenate protein. Similarly, $65-$ $70 \%$ of PTP1B protein was immunoprecipitated by $5 \mu \mathrm{g}$ of our polyclonal antibody, and the commercially available LRP and SH-PTP2 antibodies (Transduction Laboratories, Lexington, NY) deplete 80$85 \%$ of the LRP or SH-PTP2 content from the tissue particulate fraction.

Statistical methods. Data are presented as mean \pm SEM with the indicated number of samples in each group. Correlations were analyzed by linear regression analysis, and where applicable, two groups were compared by Student's $t$ test, and three or more groups were analyzed by ANOVA with Bonferroni's correction to evaluate the significance of the observed differences. Calculations were performed (SigmaStat PC computing software; Jandel Scientific, San Rafael, CA).

\section{Results}

Human subjects. Skeletal muscle was obtained during elective surgery from six normal weight (lean) women, five obese women without diabetes, and four obese women with NIDDM (Table I). The mean age of the three groups did not differ. The mean BMI values from the two groups of obese patients were significantly different from the lean group. The mean plasma glucose values of the nondiabetic obese group were not different from the lean controls, while the glucoses of the obese subjects with NIDDM were significantly elevated. As a marker of physiological insulin resistance, the plasma insulin levels were elevated significantly in both groups of obese subjects, with the highest level in the diabetic subjects (Table I).

Glucose uptake into skeletal muscle in vitro. Insulin-stimulated uptake of 2-deoxyglucose was also measured to evaluate

Table I. Characteristics of the Study Patients

\begin{tabular}{lccccc}
\hline Patient group & $n$ & Age & BMI & Glucose & Insulin \\
\hline & & $y r$ & $k g / m^{2}$ & $m g / d l$ & $\mu U / m l$ \\
Lean & 6 & $39.3 \pm 1.4$ & $21.9 \pm 1.2$ & $84 \pm 4$ & $2.1 \pm 0.4$ \\
Obese & 5 & $43.4 \pm 3.9$ & $39.8 \pm 2.9^{*}$ & $95 \pm 5$ & $8.7 \pm 2.3^{*}$ \\
Diabetic & 4 & $46.5 \pm 2.0$ & $51.5 \pm 8.4^{*}$ & $146 \pm 28^{*}$ & $17.9 \pm 3.8^{*}$
\end{tabular}

The BMI is the weight in kilograms divided by the square of the height in meters. The data are presented as mean \pm standard error. ${ }^{*} P<0.05$ compared to the lean group.

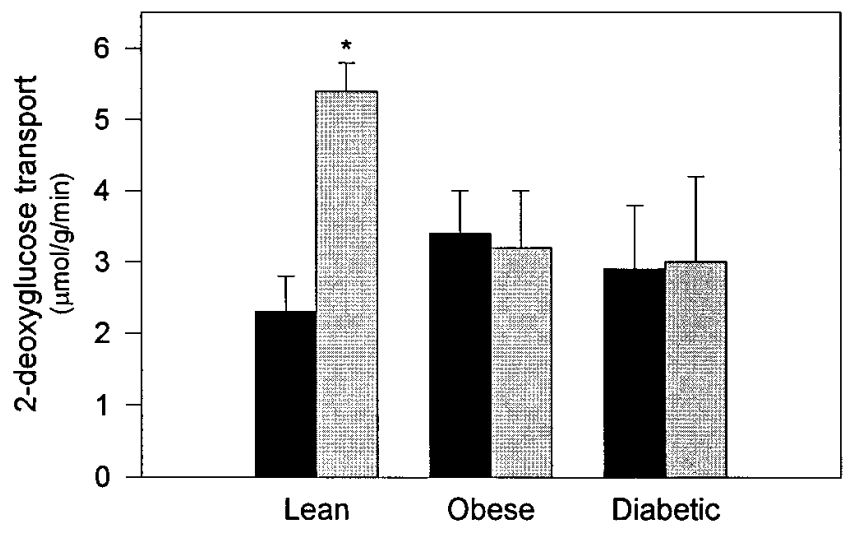

Figure 1. 2-Deoxyglucose transport in muscle fiber strips from nonobese subjects, obese subjects, and obese subjects with NIDDM. Muscle samples from each of the control (lean), obese, and obese NIDDM subjects as indicated in Table I were isolated as described in Methods. Muscle fiber strips were prepared, and rates of glucose transport were measured under basal conditions ( 0 insulin) and after $60 \mathrm{~min}$ of maximal insulin stimulation $(100 \mathrm{nM})$, and reported as $\mu \mathrm{mol} / \mathrm{g}$ dry weight of tissue per min as described previously (27). The error bars indicate the SE for the mean values shown. $* P<0.05$ by Student's $t$ test between the basal and insulin-stimulated samples. Insulin, shaded bar; basal, black bar.

insulin action directly in the skeletal muscle strips from the study patients (Fig. 1). Skeletal muscle strips from the lean individuals showed a 2.3-fold increase in glucose uptake after insulin stimulation. There was no significant difference in the level of basal glucose transport among the three groups. The skeletal muscle strips from both of the obese groups failed to respond to insulin treatment, demonstrating a significant resistance to insulin action at the tissue level.

Skeletal muscle PTPase activities. Skeletal muscle samples were fractionated to obtain a soluble cytosol and a solubilized particulate fraction that were tested for PTPase activity with the insulin receptor and derivatized lysozyme as substrates. Since the PTPase assay was performed with matching amounts of protein from each sample, the results reflect the specific activity of the cytosol or particulate fraction PTPase activity towards the indicated substrate. A representative experiment showed that the dephosphorylation of the insulin receptor was increased markedly when incubated with either the skeletal muscle cytosol or particulate fraction from the obese individuals (Fig. 2). As a group, the PTPase activities in both subcellular fractions from the nondiabetic obese subjects were increased to $135-137 \%$ and $147-167 \%$ of the level in lean controls when tested against the insulin receptor and RCMlysozyme, respectively $(P<0.05)$ (Fig. $2 C)$. As shown in Fig. 3 , the association between BMI and the PTPase activity in the particulate fraction from the lean and obese subjects also demonstrated a significant linear correlation $(R=0.86 ; P=$ 0.0007).

In contrast, skeletal muscle PTPase activity from both the cytosol or the solubilized particulate fraction from the obese subjects with NIDDM demonstrated a significantly decreased level of PTPase activity when assayed with either the insulin receptor or RCM-lysozyme (Fig. 2). When quantitated as a group, the PTPase activities in both subcellular fractions from 

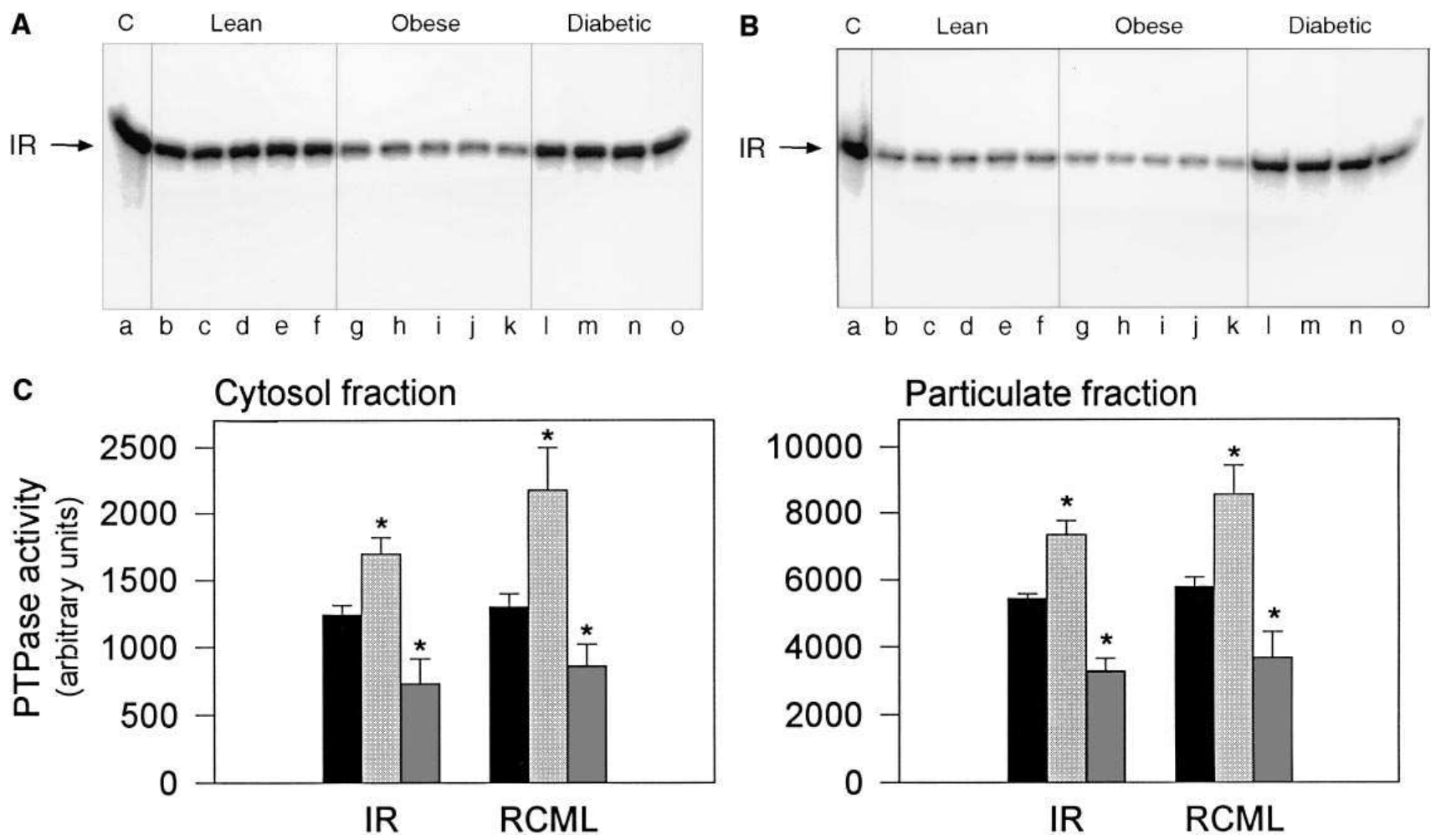

Figure 2. Insulin receptor dephosphorylation by skeletal muscle subcellular fractions from nonobese subjects, obese subjects, and obese subjects with NIDDM. Cytosol and solubilized particulate fractions were prepared from intraoperative specimens of skeletal muscle from each subject, and PTPase activity was assayed by incubation with autophosphorylated, recombinant human insulin receptors as described in Methods. A representative phosphorimage of the dephosphorylated insulin receptors is shown. Lane $a$, control incubation with no muscle fraction added; lanes $b-f$, lean subjects; lanes $g-k$, obese subjects; lanes $l-o$, obese subjects with NIDDM. ( $A$ ) Soluble cytosol fraction of skeletal muscle; $(B)$ solubilized particulate fraction of skeletal muscle. $I R$, insulin receptor kinase domain (95 kD $\beta$-subunit). (C) Quantitation of PTPase activities against the insulin receptor and RCM-lysozyme in subcellular fractions from nonobese subjects, obese subjects, and obese subjects with NIDDM. Data tabulated from PhosphorImager analysis of insulin receptor dephosphorylation shown in $A$ and $B$ are presented as a bar graph and designated with the label IR. PTPase activity was also assessed for each fraction with the labeled RCM-lysozyme substrate as described in Methods; these data are indicated by the label RCML. The error bars indicate the SE for the mean values shown. $* P<0.05$ by ANOVA for each cluster of samples from the three groups. Lean, black bars; obese, light gray bars; diabetic, dark gray bars.

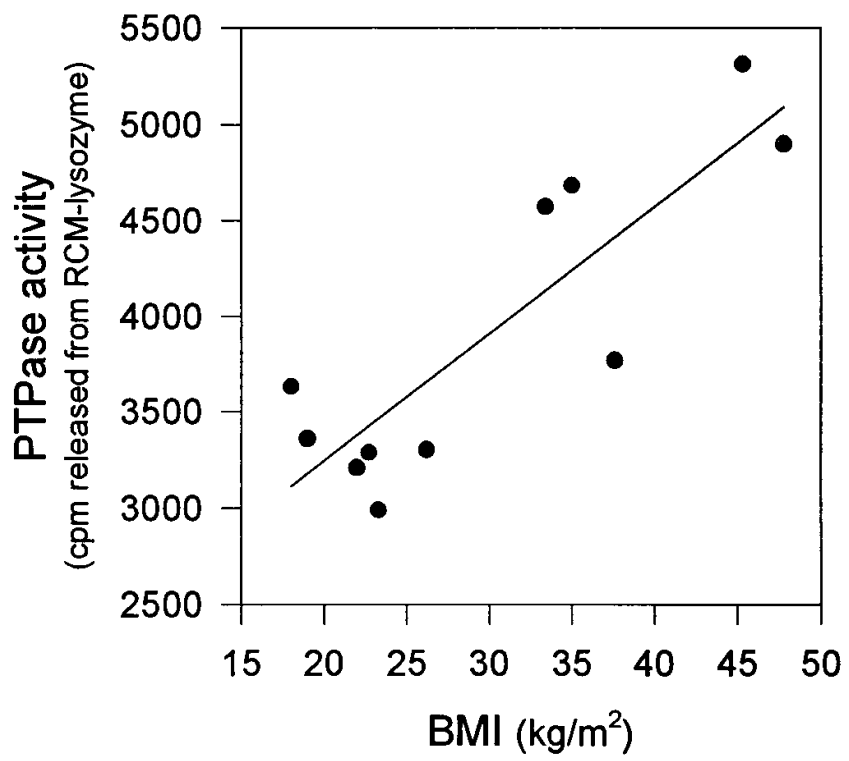

Figure 3. Correlation between PTPase activity in the particulate fraction, and BMI in the lean and obese subjects. Data from the dephos- the obese subjects with NIDDM were decreased to $39-60 \%$ and $63-66 \%$ of the level found in the lean controls when tested against the insulin receptor and RCM-lysozyme, respectively $(P<0.05)$. In addition to the comparison with lean controls, it is interesting to note the striking decrease in PTPase activity in the obese subjects with NIDDM compared to the obese nondiabetic individuals, which ranges between 40 and $44 \%$ of the elevated level seen in the obese individuals without NIDDM.

Molecular sieving chromatography of skeletal muscle PTPases. With either substrate, the PTPase activity in the solubilized particulate fraction from skeletal muscle of any of the three groups of patients consistently demonstrated a 3.9-4.5fold higher specific activity than the cytosol fraction (Fig. 2). This relatively high specific activity enabled us to perform molecular sieving chromatography on the particulate fraction to

phorylation of RCM-lysozyme for the solubilized particulate fraction of each of the lean and obese subjects were plotted against the BMI, and a linear regression line was calculated (SigmaStat; Jandel Scientific). Statistical analysis of the regression parameters revealed $R=$ 0.86 with $P=0.0007$. 


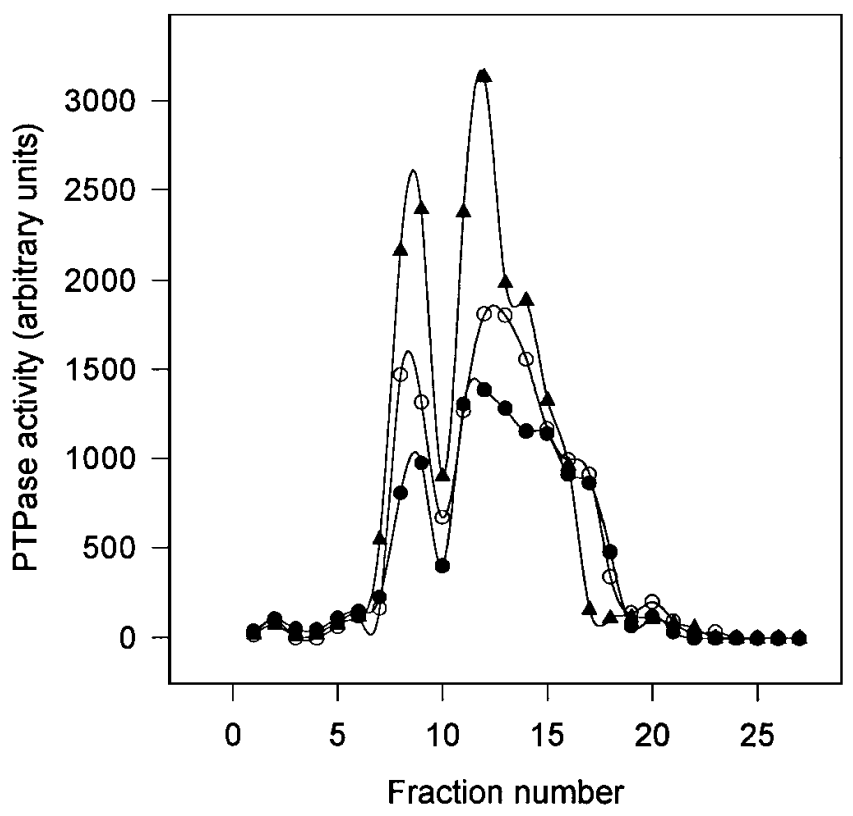

Figure 4. Molecular sieving analysis of solubilized particulate skeletal muscle PTPases from nonobese subjects, obese subjects, and obese subjects with NIDDM. As described in Methods, $7.5-\mathrm{mg}$ samples of solubilized particulate fraction protein were subjected to FPLC chromatography. Fractions were analyzed for PTPase activity using RCM-lysozyme as substrate. Lean, open circles; obese, filled triangles; diabetic, closed circles.

assess whether there were any differences in PTPases of a certain molecular size that could account for the observed changes in total PTPase in this fraction. FPLC of the solubilized particulate fraction from each of the subjects tested demonstrated two relatively sharp peaks, corresponding to relative molecular mass values of $\sim 160,000$ and $\sim 80,000 \mathrm{D}$, respectively, followed by a complex trailing peak with a shoulder (Fig. 4). Analysis of gel filtration profiles of PTPase activity from the solubilized particulate fraction from obese nondiabetic and NIDDM subjects confirmed our results indicated above that for each fraction tested, the nondiabetic obese subjects had a higher PTPase specific activity than the lean samples, while the obese NIDDM subjects demonstrated lower PTPase levels than the lean group. Furthermore, there were no striking differences in the overall profile of PTPase activity from the gel filtration analysis, indicating that the changes among the three groups involved multiple PTPase enzymes, or complexes of individual PTPase enzymes that were altered in a similar fashion.

Quantitation of specific PTPases in the soluble muscle cytosol fraction. In previous work, we demonstrated that $\mathrm{PTP} 1 \mathrm{~B}$ and SH-PTP2 are present in the soluble muscle cytosol, and are distributed between the cytosol and the particulate fraction of muscle (33). To determine whether changes in these enzymes might account for the alterations noted above in PTPase activity in the cytosol fraction from the obese and diabetic subjects, we performed immunoblotting with a protein lysate of muscle cytosol using specific antibodies to PTP1B and SHPTP2 (Fig. 5). In muscle cytosol from obese, nondiabetic subjects, PTP1B and SH-PTP2 were both increased by 1.9- and 2.0-fold respectively, compared to the lean individuals. In contrast, in the obese subjects with NIDDM the abundance of PTP1B and SH-PTP2 was significantly decreased to 34 and $51 \%$ of the value observed in the lean controls. Thus, both of these cytosolic PTPases may participate in the altered skeletal muscle PTPase activity exhibited by the obese nondiabetic subjects as well as those with NIDDM.

Quantitation of specific PTPases in the solubilized muscle particulate fraction. The particulate fraction of skeletal muscle contains high levels of the transmembrane PTPase enzymes LAR and LRP, as well as the intracellular enzymes PTP1B and SH-PTP2 (33). To determine whether the abundance of these enzymes may be altered in the obese subjects with and without NIDDM, we performed immunoblotting with samples of solubilized skeletal muscle proteins using specific antibodies to these four PTPases (Fig. 6). In the particulate fraction from obese, nondiabetic subjects, LAR and PTP1B demonstrated
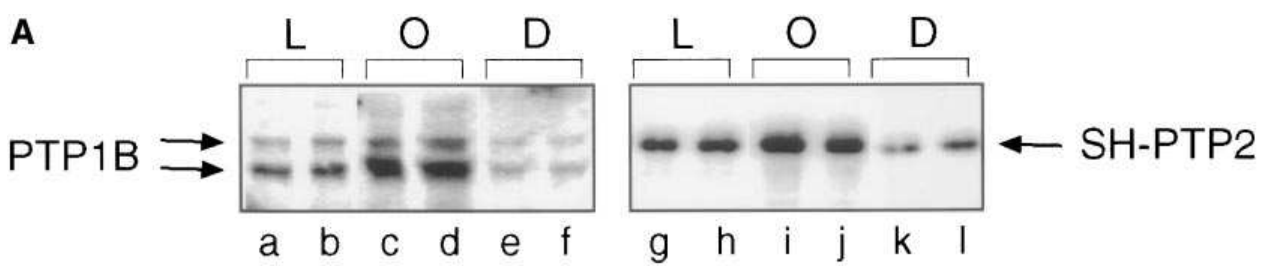

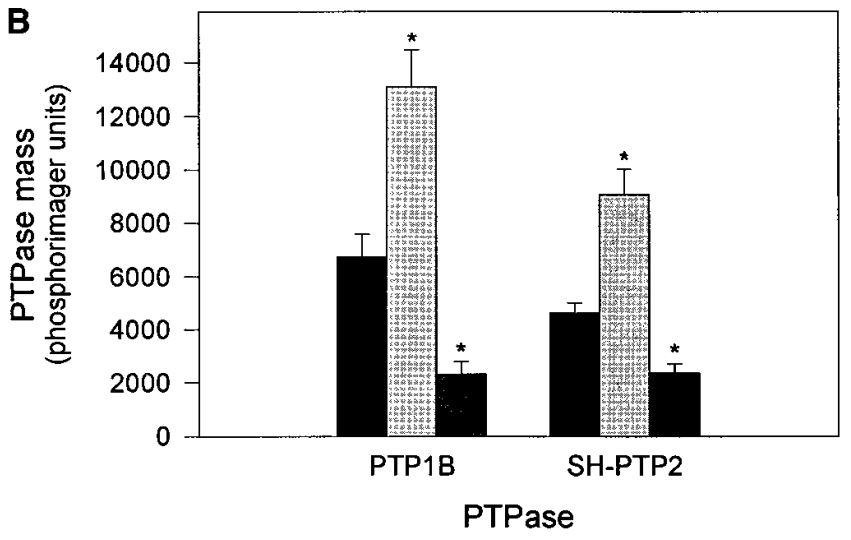

Figure 5. Abundance of PTP1B and SH-PTP2 in the cytosol fraction of skeletal muscle from the study subjects. ( $A$ ) Immunoblot analysis of PTP1B (lanes $a-f$ ) and SH-PTP2 (lanes $g-l$ ) protein abundance in representative duplicate samples from lean $(L)$, obese $(O)$, and obese NIDDM $(D)$ subjects. PTP1B is identified as two protein bands consisting of the full-length form $(\sim 50 \mathrm{kD})$ and the truncated form $(\sim 37$ $\mathrm{kD}$ ) that lacks a COOH-terminal segment (37). SH-PTP2 migrates as a single protein of $\sim 67 \mathrm{kD}$. (B) Quantitation of PTP1B and SH-PTP2 abundance in the cytosol fraction from lean, obese, and obese NIDDM subjects. Data obtained from PhosphorImager analysis of PTPase immunoblots on all of the patients in each group were tabulated and presented here in bar graph format. The error bars indicate the SE for the mean values shown. $* P<0.05$ by ANOVA for each cluster of samples from the three groups. Lean, black bars; obese, light gray bars; diabetic, dark gray bars. 

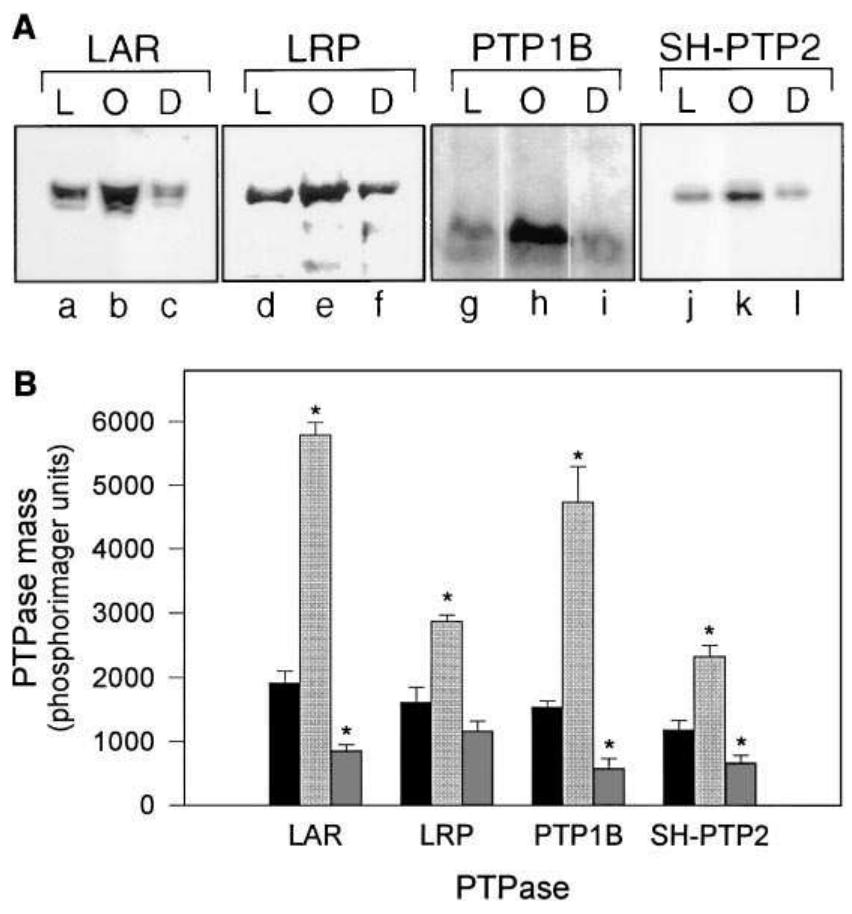

Figure 6. Abundance of LAR, LRP, PTP1B, and SH-PTP2 in the solubilized particulate fraction of skeletal muscle from the study subjects. (A) Immunoblot analysis of LAR $(a-c), \operatorname{LRP}(d-f)$, PTP1B $(g-i)$, and SH-PTP2 $(j-l)$ protein abundance in representative samples from the lean $(L)$, obese $(O)$, and obese NIDDM $(D)$ subjects. The transmembrane, or P subunit of LAR migrates at $\sim 85 \mathrm{kD}$, and LRP is identified as a single protein at $\sim 125 \mathrm{kD}$ (37). PTP1B and SH-PTP2 appear as described in the legend to Fig. 5. The full-length form of PTP1B is more prominent as would be expected in the particulate fraction (37). (B) Quantitation of LAR, LRP, PTP1B, and SH-PTP2 abundance in the solubilized particulate fraction from lean, obese, and obese NIDDM subjects. Data obtained from PhosphorImager analysis of PTPase immunoblots all of the patients in each group were tabulated and presented in bar graph format. The error bars indicate the SE for the mean values shown. $* P<0.05$ by ANOVA for each cluster of samples from the three groups. Lean, black bars; obese, light gray bars; diabetic, dark gray bars.

the greatest increase over the level observed in the lean controls; 3.0- and 3.1-fold, respectively. LRP and SH-PTP2 were also increased in the obese individuals, but less markedly, by 1.8- and 2.0-fold, respectively. As observed for the overall PTPase activity in the particulate fraction, the abundance of each of the PTPases tested was decreased in the samples from the obese subjects with NIDDM compared to the level in lean controls. The greatest change occurred with LAR and PTP1B, which were decreased to 44 and $37 \%$ of the abundance in the lean individuals, respectively. A smaller decrease, to $56 \%$ of the control level, was observed for SH-PTP2; the slight decrease observed for LRP did not reach statistical significance. These data indicated that in the particulate fraction, which contains the highest specific PTPase enzyme activity, alterations in the abundance of LAR and PTP1B correlated with the observed changes in overall tissue PTPase activity.

Immunodepletion of PTPases from skeletal muscle fractions. To further establish which of the several enzymes tested could account for the increased tissue PTPase activity in the skeletal muscle fractions from obese subjects, we used neutralizing antibodies to immunoprecipitate and deplete specific PTPase enzymes, and measured the PTPase activity remaining in the immunoprecipitation supernatant. As we have reported in previous work (23), under the immunoprecipitation conditions, the excess LAR, LRP, and SH-PTP2 antibody removed $80-90 \%$ of the individual enzymes, and the PTP-1B antibody removed $65-70 \%$ of the PTP1B present in the initial subcellular fractions.

Depletion of LAR, LRP, PTP-1B, and SH-PTP2 reduced the PTPase activity against RCM-lysozyme in the particulate enzyme samples of the control subjects to $71,73,85$, and $80 \%$, respectively, of the level observed after mock immunodepletion with nonimmune $\mathrm{IgG}$, indicating that each of these PTPases contributes substantially to the total activity observed in the solubilized particulate fraction (Fig. 7). In the particulate fraction from obese subjects, depletion of LAR, LRP, PTP-1B, and SH-PTP2 reduced the PTPase activity against RCMlysozyme to $61,84,90$, and $83 \%$, respectively, of the level observed after mock immunodepletion with nonimmune $\mathrm{IgG}$. The most significant change was the reduced PTPase activity in the sample immunodepleted of LAR; this was the only maneuver that normalized the difference between the samples from control subjects and the elevated level observed in the obese nondiabetic subjects. After immunodepletion of any of the other PTPases, the statistical significance of the quantitative difference between the lean controls and the obese subjects was maintained (Fig. 7).

The results from the LAR immunodepletion experiment with the RCM-lysozyme substrate were confirmed using the autophosphorylated insulin receptor as a PTPase substrate and the solubilized particulate fraction from each group (Fig. 8). After mock immunodepletion with control $\mathrm{IgG}$, insulin receptor dephosphorylation with samples from the obese subjects was increased by $32 \%$ over the level in lean controls, and the re-

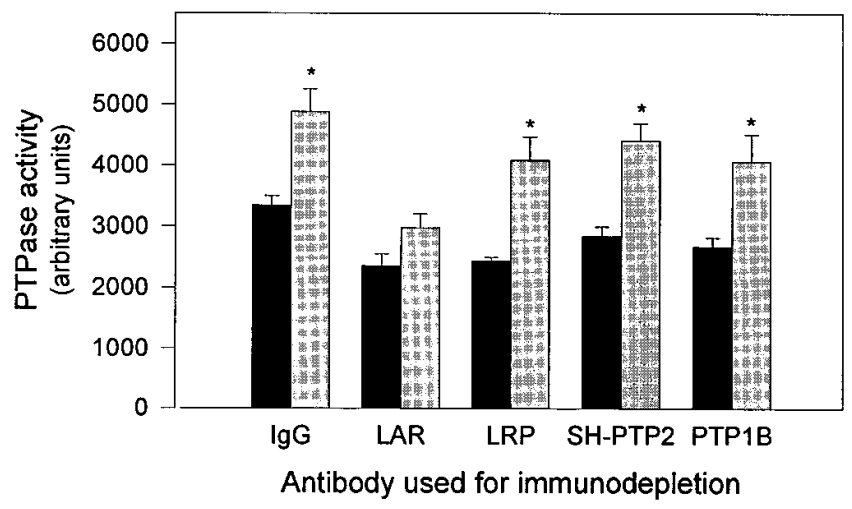

Figure 7. PTPase activity remaining in the solubilized particulate fraction of skeletal muscle from the study subjects after immunodepletion with the indicated PTPase antibodies. Aliquots of the particulate fractions were incubated with excess antibody to each of the four PTPases as indicated or with normal rabbit IgG, and immunocomplexes were isolated on Trisacryl-protein A beads to deplete the supernatant of the enzymes as described in Methods. The PTPase activity remaining was determined with the RCM-lysozyme substrate. The error bars indicate the $\mathrm{SE}$ for the mean values shown. $* P<0.05$ by Student's $t$ test between the samples from lean controls or obese subjects. Lean, black bars; obese, light gray bars. 


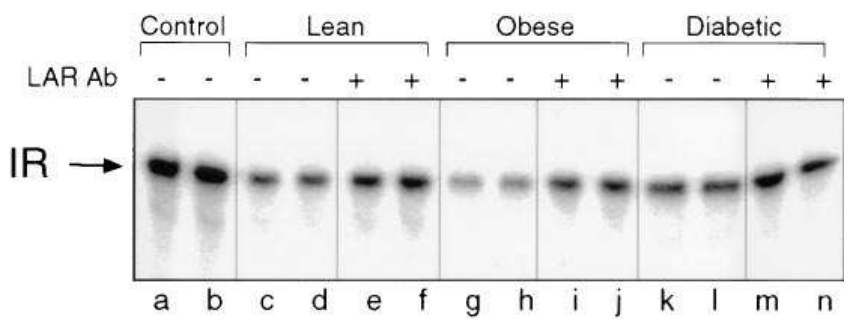

Figure 8. PTPase activity against the insulin receptor remaining in the solubilized particulate fraction of skeletal muscle from the study subjects after immunodepletion with LAR antibodies. Aliquots of the particulate fractions were incubated with excess LAR antibody or with normal rabbit IgG as indicated in duplicate. The immunocomplexes were isolated on Trisacryl-protein A beads to deplete the supernatant of the enzymes as described in Methods. The remaining PTPase activity was determined with the autophosphorylated insulin receptor as described in Methods. A representation phosphorimage of the dephosphorylated insulin receptors is shown. $a-b$, Control incubation with no muscle fraction added; $c-f$, lean subjects; $g-j$, obese subjects; $k-n$, obese subjects with NIDDM. $I R$, insulin receptor kinase domain ( $95 \mathrm{kD} \beta$-subunit).

ceptor dephosphorylation by the subjects with NIDDM was decreased to $78 \%$ of the control value, similar to the changes noted above in Fig. $2 \mathrm{~B}$. After LAR immunodepletion, the samples from lean, obese, and NIDDM subjects were reduced to 78,64 , and $71 \%$ of the level observed after mock depletion (Fig. 8). This result indicated that in each of the samples, LAR, or a closely related PTPase susceptible to immunodepletion with the anti-LAR antibodies, comprises a significant fraction of the insulin receptor dephosphorylating activity in the particulate fraction. Furthermore, these results confirmed that after immunodepletion with the LAR antibodies, the difference in insulin receptor dephosphorylation between the lean and

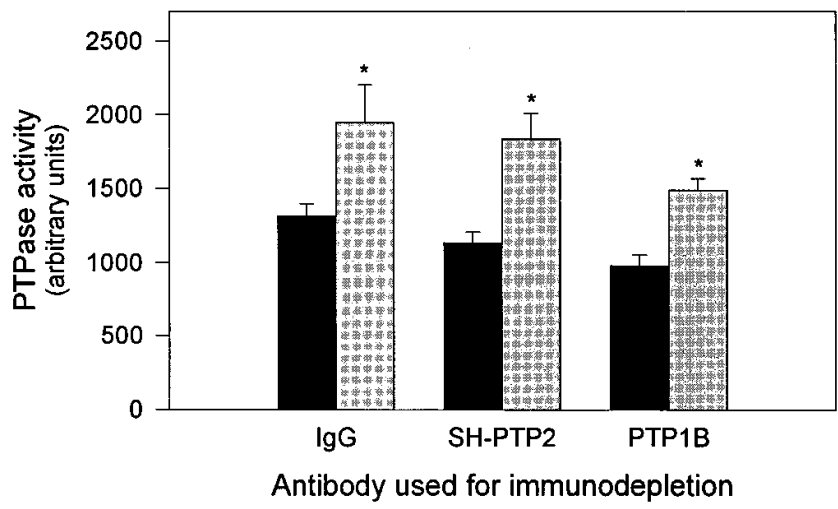

Figure 9. PTPase activity remaining in the cytosol fraction of skeletal muscle from the study subjects after immunodepletion with antibodies to either SH-PTP2 or PTP1B. Aliquots of the cytosol fractions were incubated with normal rabbit IgG or with excess antibody to the indicated PTPases, and immunocomplexes were isolated on Trisacryl-protein A beads to deplete the supernatant as described in Methods. The remaining PTPase activity was determined with the RCM-lysozyme substrate. The error bars indicate the SE for the mean values shown. ${ }^{*} P<0.05$ by Student's $t$ test between the samples from lean controls or obese subjects. obese subjects was eliminated; the level of insulin receptor dephosphorylation for equivalent amounts of particulate fraction protein were 50 and $54 \%$ for the lean and obese samples, respectively. These findings also supported the conclusion that the increased receptor dephosphorylating activity in the particulate fraction was largely due to an increase in LAR or a closely related PTPase.

In the cytosol fraction of the lean subjects, immunodepletion of PTP-1B and SH-PTP2 reduced the PTPase activity against RCM-lysozyme to 87 and $74 \%$, respectively, of the level observed after the control incubation with nonimmune IgG, indicating that these PTPases contribute to the overall PTPase activity observed in the skeletal muscle cytosol fraction (Fig. 9). In the cytosol fraction from obese subjects, depletion of PTP-1B and SH-PTP2 reduced the PTPase activity against RCM-lysozyme to 94 and $77 \%$, respectively, of the level observed after mock immunodepletion with nonimmune IgG. Moreover, the immunodepleted samples from the obese subjects retained a significant difference when compared to the lean control subjects, suggesting that neither PTP1B nor SH-PTP2 alone could account for the increase in cytosol PTPase activity in the skeletal muscle samples from obese subjects.

\section{Discussion}

Various mechanisms that can potentially influence postbinding events in insulin signaling have been hypothesized to cause the insulin resistance observed in skeletal muscles in human obesity and NIDDM (34-36). One mechanism that has been receiving increasing attention involves modulation of the steadystate level of tyrosine phosphorylation of the insulin receptor and its cellular substrate proteins by PTPases (37). The recent discovery of a family of PTPase enzymes expressed in insulinsensitive tissues has provided the basis for detailed studies to examine the role that these enzymes play in the regulation of reversible tyrosine phosphorylation in insulin receptor signaling as well as their potential involvement in insulin-resistant disease states.

In the present work, we have extended our previous finding in adipose tissue that PTPase activity and the abundance of specific PTPase enzymes is increased in obese, insulin-resistant subjects (23). We now find a similar result in skeletal muscle, that PTPase activity against the insulin receptor in the particulate and soluble cytosol subcellular fractions of muscle from nondiabetic obese subjects was increased significantly by 40 $70 \%$ compared to the level in lean controls. Also, similar to our results in adipose tissue, the increased PTPase activity in the skeletal muscle particulate fraction appeared to be primarily due to an increase in LAR or a closely related PTPase, since immunodepletion of this PTPase normalized the elevated PTPase activity in the solubilized particulate fraction towards the insulin receptor (Fig. 7). These data are supported by previous work by McGuire et al. (38), who reported that skeletal muscle from insulin-resistant Pima Native American subjects had a $33 \%$ higher basal PTPase activity towards phosphorylated RCM-lysozyme in the particulate fraction compared to insulinsensitive controls. Furthermore, insulin infusion in vivo produced a rapid $25 \%$ suppression of soluble PTPase activity in muscle of insulin-sensitive subjects, a response that was impaired severely in the insulin-resistant subjects, suggesting abnormal regulation of muscle PTPases (38). Together, these data provide evidence for the hypothesis that increased PTP- 
ase activity, in particular involving LAR, may play an important role in insulin resistance in both skeletal muscle and adipose tissue in insulin-resistant states.

The observation that LAR is increased in human obesity is of interest, since evidence from a variety of experimental approaches has suggested that this widely expressed transmembrane PTPase is a major candidate for the physiological regulation of the insulin receptor $(5,37)$. In addition to its tissue distribution and membrane localization, biochemical studies have demonstrated that the cytoplasmic domain of LAR has a catalytic preference for the dephosphorylation of regulatory phosphotyrosines in the insulin receptor, and it can efficiently deactivate the receptor kinase activity (30). In transfection studies, LAR has been shown to act as a negative regulator of insulin signaling in intact cells. These experiments have included the use of LAR antisense mRNA expression in hepatoma cells to reduce the LAR mass, which significantly enhances insulin receptor autophosphorylation, receptor kinase activity, and insulin-stimulated phosphatidylinositol 3'-kinase activity (16). In addition, overexpression of the full-length LAR protein at the plasma membrane in both hepatoma cells and $\mathrm{CHO}$ cells significantly reduces insulin receptor autophosphorylation and kinase activity in situ $(13,14)$. Recently, we have also demonstrated a physical association between LAR and the insulin receptor by coimmunoprecipitation from cell lysates and using chemical cross-linking techniques (39). Overall, the available evidence suggests that the functional effects of LAR on insulin signaling are likely to be mediated by a direct interaction with the insulin receptor.

Additional PTPases may also be involved in the regulation of the insulin action pathway. When microinjected into oocytes, PTP1B, a widely expressed, single domain intracellular PTPase, blocked insulin-stimulated S6 peptide phosphorylation, and retarded insulin-induced oocyte maturation $(40,41)$. In cultured cells, transfection of PTPase cDNAs has also shown that $\mathrm{PTP} 1 \mathrm{~B}$ can regulate negatively receptors for insulin as well as IGF-1, PDGF, and EGF (19). We have shown by osmotic loading of neutralizing antibodies into insulin-sensitive hepatoma cells, that PTP1B negatively regulates insulin receptor activation (18). Since PTP1B has a role in the negative regulation of insulin signaling and acts, at least in part, directly at the level of the receptor kinase, it may function in concert with LAR in the physiological regulation of the insulin receptor. Other PTPases have been implicated in a few studies as negative regulators of insulin action, including LRP and the closely related transmembrane enzyme RPTP- (15). In contrast, SHPTP2, a widely expressed homolog with two $\mathrm{NH}_{2}$-terminal $\mathrm{SH} 2$ domains, has been implicated as a positive mediator of insulin signaling as a downstream site $(21,42,43)$.

One of the most striking results in our study was that the obese subjects with NIDDM exhibited a significantly decreased level of skeletal muscle PTPase activity in both soluble and particulate subcellular fractions, to as low as 39\% of the level found in the lean controls. This result suggests that a major alteration in this regulatory system occurs in obese individuals after the onset of diabetes with overt hyperglycemia. Alternatively, the subjects with NIDDM may be inherently different from the obese nondiabetic group, with an altogether different pathophysiologic mechanism that may account for their underlying insulin resistance. A few recent studies have also suggested that skeletal muscle PTPase activity might be decreased in patients with NIDDM. Worm et al. (44) noted a marked decrease in soluble PTPase activity in skeletal muscle biopsies from patients with NIDDM, to $42 \%$ of the activity in the control subjects. In the study by Kusari et al. (45), skeletal muscle particulate fraction PTPase activity against RCMlysozyme was also reduced by $\sim 20 \%$ in subjects with NIDDM; this was associated with an average decrease of $38 \%$ in the abundance of PTP1B protein abundance in the diabetic subjects, similar to our results reported here.

Kusari et al. (45) also reported a decrease in PTPase activity in their sample of obese, nondiabetic subjects, although their patient population and assay methodology differed from that reported in the present work in several respects. Their obese subjects represented a different population since they were only moderately overweight (mean BMI 33.0), a factor that may have obscured detection of increased PTPase activity in subjects with BMI values as high as those of the morbidly obese individuals in our population. In addition, the PTPase assay for the obese subjects in the study by Kusari et al. (45) used a phosphorylated peptide derived from the kinase domain of the insulin receptor. The use of this peptide substrate may cause confusing results because the level of tissue PTPase activity detected depends heavily on the phosphotyrosyl form of the peptide (46). Thus, their results for obese nondiabetic subjects may not be comparable to our data obtained using the intact insulin receptor protein as substrate, or RCM-lysozyme. Furthermore, in their work, the abundance of protein mass for PTP1B was measured only in the subjects with NIDDM and not the obese nondiabetic individuals as we have reported here, so our two studies cannot be compared directly in this regard.

Alterations in tissue PTPases similar to those reported in the present work have been noted in some diabetic animal models. Particulate fraction PTPase activity towards the insulin receptor or artificial substrates has been shown in several studies to be decreased significantly in livers of insulin-deficient rats generated by streptozotocin $(47,48)$ or alloxan $(49)$ treatment, with a recovery to normal levels after insulin injections. In our own work, we have also found that the particulate fraction PTPase activity towards the insulin receptor in streptozotocin-treated rats was decreased to $65-70 \%$ of control in diabetic liver and muscle, and increased to $115-120 \%$ of control after insulin treatment (50).

The results in subjects with NIDDM suggest that in states of relative insulin deficiency, or with defective insulin action, in both human and animal studies, PTPase activity in muscle may be diminished. These findings raise the question whether the observed alterations in PTPase abundance are primary or may result secondarily from the deranged metabolic milieu in longstanding obesity or frank diabetes. For example, an increase in PTPase activity that negatively regulates the insulin signaling system, as we have observed in adipose tissue and skeletal muscle, may be causally related to insulin resistance in simple obesity without diabetes, and may occur as an initial defect that leads to the hyperinsulinemia. Alternatively, hyperinsulinemia from tissue insulin resistance caused by an unrelated defect may lead to increased PTPase abundance in skeletal muscle and adipose tissue. In this regard, insulin infusion has been shown to modulate tissue PTPase activities in normal subjects, and that this regulation may be disrupted in insulin-resistant or diabetic individuals $(38,44)$. Insulin has been shown to increase significantly the particulate fraction PTPase activity in rat hepatoma cells $(51,52)$ and in rat L6 muscle cells $(53)$. 
In summary, these studies lend further support to the hypothesis that specific PTPases are increased in skeletal muscle in human obesity, and may play an important role in the pathogenesis of insulin resistance in this tissue, similar to the findings we have reported previously for adipose tissue. Interestingly, we also provide evidence for reduced expression of PTPases in skeletal muscle in individuals with NIDDM, as has been reported in previous work from other laboratories. The clear distinction in the level of tissue PTPase activities in our obese subjects compared to those with NIDDM suggests that this effect may have an entirely different pathophysiological basis in these two groups of individuals. Such divergent regulatory mechanisms may differentially lead to insulin resistance in obesity, or to NIDDM in susceptible individuals. It remains to be determined whether the increased PTPase activity in obesity is a primary alteration that leads to insulin resistance, or results secondarily from a metabolic alteration that occurs with obesity and contributes to the insulin-resistant state. In a similar fashion, the decreased PTPase activity observed in NIDDM may be part of the underlying pathogenesis of this entity, which might be expected to impact on insulin signaling through the development of the diabetes. Alternatively, a decrease in PTPase activity may occur as a secondary response to the diabetic milieu. In fact, incubation of insulin-resistant skeletal muscle from nondiabetic obese subjects in a high glucose medium leads to enhanced insulin responsiveness towards several aspects of glucose disposal, including glycogen synthesis, glucose oxidation, and nonoxidative glycolysis (54). The possibility that elevated glucose leads to enhanced insulin signaling by reducing PTPase expression in muscle from obese subjects remains to be determined.

Further studies in skeletal muscle and adipose tissue from obese subjects and patients with NIDDM in which glucose and insulin are regulated, as well as additional work in cultured human adipocytes and skeletal muscle strips, will be necessary to elucidate further the mechanism of altered PTPase activity and changes in the abundance of specific PTPase enzymes as we have observed in this work. It will also be important to determine whether the increased PTPase levels in skeletal muscle in the nondiabetic obese subjects are reversed after weight loss, as we have observed for PTPases in adipose tissue, adding further support to the hypothesis that they have an important role in the tissue insulin resistance observed in this group of subjects.

\section{Acknowledgments}

These studies were supported by National Institutes of Health grants R01 DK43396 and 45592 to Dr. Goldstein.

\section{References}

1. Rabinowitz, D. 1970. Some endocrine and metabolic aspects of obesity. Annu. Rev. Med. 21:241-258.

2. Kolterman, O.G., J. Insel, M. Saekow, and J.M. Olefsky. 1980. Mechanisms of insulin resistance in human obesity: evidence for receptor and postreceptor defects. J. Clin. Invest. 65:1272-1284.

3. Caro, J.F. 1991. Clinical Review 26: insulin resistance in obese and nonobese man. J. Clin. Endocrinol. Metab. 73:691-695.

4. White, M.F., and C.R. Kahn. 1994. The insulin signaling system. J. Biol. Chem. 269:1-4.

5. Goldstein, B.J. 1993. Regulation of insulin receptor signaling by proteintyrosine dephosphorylation. Receptor. 3:1-15.

6. Fischer, E.H., H. Charbonneau, and N.K. Tonks. 1991. Protein tyrosine phosphatases: a diverse family of intracellular and transmembrane enzymes. Science (Wash. DC). 253:401-406.

7. Streuli, M., N.X. Krueger, L.R. Hall, S.F. Schlossman, and H. Saito. 1988. A new member of the immunoglobulin superfamily that has a cytoplasmic region homologous to the leukocyte common antigen. J. Exp. Med. 168:15231530 .

8. Sap, J., P. D'Eustachio, D. Givol, and J. Schlessinger. 1990. Cloning and expression of a widely expressed receptor tyrosine phosphatase. Proc. Natl. Acad. Sci. USA. 87:6112-6116.

9. Chernoff, J., A.R. Schievella, C.A. Jost, R.L. Erikson, and B.G. Neel. 1990. Cloning of a cDNA for a major human protein-tyrosine-phosphatase. Proc. Natl. Acad. Sci. USA. 87:2735-2739.

10. Guan, K.L., R.S. Haun, S.J. Watson, R.L. Geahlen, and J.E. Dixon. 1990. Cloning and expression of a protein-tyrosine-phosphatase. Proc. Natl. Acad. Sci. USA. 87:1501-1505.

11. Freeman, R.M., J. Plutzky, and B.G. Neel. 1992. Identification of a human src homology 2-containing protein-tyrosine-phosphatase: a putative homolog of drosophila corkscrew. Proc. Natl. Acad. Sci. USA. 89:11239-11243.

12. Ding, W., W.R. Zhang, K. Sullivan, N. Hashimoto, and B.J. Goldstein. 1994. Identification of protein-tyrosine phosphatases prevalent in adipocytes by molecular cloning. Biochem. Biophys. Res. Commun. 202:902-907.

13. Li, P.M., W.R. Zhang, and B.J. Goldstein. 1996. Suppression of insulin receptor activation by overexpression of the protein-tyrosine phosphatase LAR in hepatoma cells. Cell. Signaling. 8:467-473.

14. Zhang, W.R., P.M. Li, M.A. Oswald, and B.J. Goldstein. 1996. Modulation of insulin signal transduction by eutopic overexpression of the receptortype protein-tyrosine phosphatase LAR. Mol. Endocrinol. 10:575-584.

15. Moller, N.P.H., K.B. Moller, R. Lammers, A. Kharitonenkov, E. Hoppe, F.C. Wiberg, I. Sures, and A. Ullrich. 1995. Selective down-regulation of the insulin receptor signal by protein-tyrosine phosphatases alpha and epsilon. J. Biol. Chem. 270:23126-23131.

16. Kulas, D.T., W.R. Zhang, B.J. Goldstein, R.W. Furlanetto, and R.A. Mooney. 1995. Insulin receptor signaling is augmented by antisense inhibition of the protein-tyrosine phosphatase LAR. J. Biol. Chem. 270:2435-2438.

17. Kenner, K.A., E. Anyanwu, J.M. Olefsky, and J. Kusari. 1996. Proteintyrosine phosphatase $1 \mathrm{~B}$ is a negative regulator of insulin- and insulin-like growth factor-1 stimulated signaling. J. Biol. Chem. 271:19810-19816.

18. Ahmad, F., P.M. Li, J. Meyerovitch, and B.J. Goldstein. 1995. Osmotic loading of neutralizing antibodies defines a role for protein-tyrosine phosphatase 1B in negative regulation of the insulin action pathway. J. Biol. Chem. 270:20503-20508

19. Lammers, R., B. Bossenmaier, D.E. Cool, N.K. Tonks, J. Schlessinger, E.H. Fischer, and A. Ullrich. 1993. Differential activities of protein tyrosine phosphatases in intact cells. J. Biol. Chem. 268:22456-22462.

20. Kuhné, M.R., T. Pawson, G.E. Lienhard, and G.S. Feng. 1993. The insulin receptor substrate-1 associates with the SH2-containing phosphotyrosine phosphatase Syp. J. Biol. Chem. 268:11479-11481.

21. Xiao, S., D.W. Rose, T. Sasaoka, H. Maegawa, T.R. Burke, P.P. Roller, S.E. Shoelson, and J.M. Olefsky. 1994. Syp (SH-PTP2) is a positive mediator of growth factor-stimulated mitogenic signal transduction. J. Biol. Chem. 269: 21244-21248.

22. Milarski, K.L., and A.R. Saltiel. 1994. Expression of catalytically inactive Syp phosphatase in 3T3 cells blocks stimulation of mitogen-activated protein kinase by insulin. J. Biol. Chem. 269:21239-21243.

23. Ahmad, F., R.V. Considine, and B.J. Goldstein. 1995. Increased abundance of the receptor-type protein-tyrosine phosphatase LAR accounts for the elevated insulin receptor dephosphorylating activity in adipose tissue of obese human subjects. J. Clin. Invest. 95:2806-2812.

24. Ahmad, F., R.V. Considine, T.L. Bauer, J.P. Ohannesian, C.C. Marco, and B.J. Goldstein. 1997. Improved sensitivity to insulin in obese subjects following weight loss is accompanied by reduced protein-tyrosine phosphatases in adipose tissue. Metab. Clin. Exp. In press.

25. DeFronzo, R.A., R.C. Bonadonna, and E. Ferrannini. 1992. Pathogenesis of NIDDM. A balanced overview. Diabetes Care. 15:318-368.

26. National Diabetes Data Group. 1979. Classification and diagnosis of diabetes mellitus and other categories of glucose intolerance. Diabetes. 28:10391057.

27. Dohm, G.L., E.B. Tapscott, W.J. Pories, D.J. Dabbs, E.G. Flickinger, D Meelheim, T. Fushiki, S.M. Atkinson, C.W. Elton, and J.F. Caro. 1988. An in vitro human muscle preparation suitable for metabolic studies. J. Clin. Invest. 82:486-494.

28. Bradford, M.M. 1976. A rapid and sensitive method for the quantitation of microgram quantities of protein utilizing the principle of protein-dye binding. Anal. Biochem. 72:248-254.

29. Kasuga, M., M.F. White, and C.R. Kahn. 1985. Phosphorylation of the insulin receptor in cultured hepatoma cells and a solubilized system. Methods Enzymol. 109:609-621.

30. Hashimoto, N., E.P. Feener, W.R. Zhang, and B.J. Goldstein. 1992. Insulin receptor protein-tyrosine phosphatases-leukocyte common antigenrelated phosphatase rapidly deactivates the insulin receptor kinase by preferential dephosphorylation of the receptor regulatory domain. J. Biol. Chem. 267: 13811-13814. 
31. Laemmli, E.K. 1970. Cleavage of structural proteins during the assembly of the head of bacteriophage $\mathrm{T}_{4}$. Nature (Lond.). 227:680-685.

32. Tonks, N.K., C.D. Diltz, and E.H. Fischer. 1991. Purification and assay of CD45: an integral membrane protein-tyrosine phosphatase. Methods Enzymol. 201:442-451.

33. Ahmad, F., and B.J. Goldstein. 1995. Purification, identification and subcellular distribution of three predominant protein-tyrosine phosphatase enzymes in skeletal muscle tissue. Biochim. Biophys. Acta. 1248:57-69.

34. Haring, H.U., and H. Mehnert. 1993. Pathogenesis of type-2 (non-insulin-dependent) diabetes-mellitus-candidates for a signal transmitter defect causing insulin resistance of the skeletal muscle. Diabetologia. 36:176-182.

35. Caro, J.F., L.G. Dohm, W.J. Pories, and M.K. Sinha. 1989. Cellular alterations in liver, skeletal muscle, and adipose tissue responsible for insulin resistance in obesity and type II diabetes. Diabetes Metab. Rev. 5:665-689.

36. Goodyear, L.J., F. Giorgino, L.A. Sherman, J. Carey, R.J. Smith, and G.L. Dohm. 1995. Insulin receptor phosphorylation, insulin receptor substrate-1 phosphorylation, and phosphatidylinositol 3-kinase activity are decreased in intact skeletal muscle strips from obese subjects. J. Clin. Invest. 95:2195-2204.

37. Goldstein, B.J. 1996. Protein-tyrosine phosphatases and the regulation of insulin action. In Diabetes Mellitus: A Fundamental and Clinical Text. D. LeRoith, J.M. Olefsky, and S.I. Taylor, editors. Lippincott Raven Publishers, Philadelphia. 174-186.

38. McGuire, M.C., R.M. Fields, B.L. Nyomba, I. Raz, C. Bogardus, N.K. Tonks, and J. Sommercorn. 1991. Abnormal regulation of protein tyrosine phosphatase activities in skeletal muscle of insulin-resistant humans. Diabetes. 40:939-942.

39. Ahmad, F., and B.J. Goldstein. 1997. Functional association between the insulin receptor and the transmembrane protein-tyrosine phosphatase LAR in intact cells. J. Biol. Chem. 272:448-457.

40. Cicirelli, M.F., N.K. Tonks, C.D. Diltz, J.E. Weiel, E.H. Fischer, and E.G. Krebs. 1990. Microinjection of a protein-tyrosine-phosphatase inhibits insulin action in Xenopus oocytes. Proc. Natl. Acad. Sci. USA. 87:5514-5518.

41. Tonks, N.K., M.F. Cicirelli, C.D. Diltz, E.G. Krebs, and E.H. Fischer. 1990. Effect of microinjection of a low-Mr human placenta protein tyrosine phosphatase on induction of meiotic cell division in Xenopus oocytes. Mol. Cell. Biol. 10:458-463.

42. Yamauchi, K., K.L. Milarski, A.R. Saltiel, and J.E. Pessin. 1995. Protein-tyrosine-phosphatase SHPTP2 is a required positive effector for insulin downstream signaling. Proc. Natl. Acad. Sci. USA. 92:664-668.

43. Noguchi, T., T. Matozaki, K. Horita, Y. Fujioka, and M. Kasuga. 1994.
Role of SH-PTP2, a protein-tyrosine phosphatase with Src homology 2 domains, in insulin-stimulated ras activation. Mol. Cell. Biol. 14:6674-6682.

44. Worm, D., J. Vinten, P. Staehr, J.E. Henriksen, A. Handberg, and H. Beck-Nielsen. 1996. Altered basal and insulin-stimulated phosphotyrosine phosphatase (PTPase) activity in skeletal muscle fractions from NIDDM patients compared with control subjects. Diabetologia. 39:1208-1214.

45. Kusari, J., K.A. Kenner, K.I. Suh, D.E. Hill, and R.R. Henry. 1994. Skeletal muscle protein tyrosine phosphatase activity and tyrosine phosphatase 1B protein content are associated with insulin action and resistance. J. Clin. Invest. 93:1156-1162.

46. Sredy, J., D.R. Sawicki, B.R. Flam, and D. Sullivan. 1995. Insulin resistance is associated with abnormal dephosphorylation of a synthetic phosphopeptide corresponding to the major autophosphorylation sites of the insulin receptor. Metab. Clin. Exp. 44:1074-1081.

47. Meyerovitch, J., J.M. Backer, and C.R. Kahn. 1989. Hepatic phosphotyrosine phosphatase activity and its alteration in diabetic rats. J. Clin. Invest. 84 976-983.

48. Hauguel deMouzon, S., P. Peraldi, F. Alengrin, and E. Van Obberghen 1993. Alteration of phosphotyrosine phosphatase activity in tissues from diabetic and pregnant rats. Endocrinology. 132:67-74.

49. Gruppuso, P.A., J.M. Boylan, B.I. Posner, R. Faure, and D.L. Brautigan. 1990. Hepatic protein phosphotyrosine phosphatase. Dephosphorylation of insulin and epidermal growth factor receptors in normal and alloxan diabetic rats. J. Clin. Invest. 85:1754-1760.

50. Ahmad, F., and B.J. Goldstein. 1995. Alterations in specific proteintyrosine phosphatases accompany the insulin resistance of streptozotocin-diabetes. Am. J. Physiol. 268:E932-E940.

51. Meyerovitch, J., J.M. Backer, P. Csermely, S.E. Shoelson, and C.R. Kahn. 1992. Insulin differentially regulates protein phosphotyrosine phosphatase activity in rat hepatoma cells. Biochemistry. 31:10338-10344.

52. Hashimoto, N., and B.J. Goldstein. 1992. Differential regulation of mRNAs encoding three protein-tyrosine phosphatases by insulin and activation of protein kinase C. Biochem. Biophys. Res. Commun. 188:1305-1311.

53. Kenner, K.A., D.E. Hill, J.M. Olefsky, and J. Kusari. 1993. Regulation of protein tyrosine phosphatases by insulin and insulin-like growth factor-I. $J$. Biol. Chem. 268:25455-25462.

54. Friedman, J.E., J.F. Caro, W.J. Pories, J.L. Azevedo, and G.L. Dohm 1994. Glucose metabolism in incubated human muscle: effect of obesity and non-insulin-dependent diabetes mellitus. Metab. Clin. Exp. 43:1047-1054. 